
allemande

49-1 | 2017

Berlin 1957-1994

\title{
Politische Feierkonkurrenz im Jahre 1987. Die doppelte 750-Jahr-Feier in Ost- und West-Berlin
}

Krijn Thijs

\section{(2) OpenEdition}

1 Journals

Édition électronique

URL : https://journals.openedition.org/allemagne/523

DOI : 10.4000/allemagne.523

ISSN : 2605-7913

Éditeur

Société d'études allemandes

Édition imprimée

Date de publication : 16 juin 2017

Pagination : 71-84

ISSN : 0035-0974

\section{Référence électronique}

Krijn Thijs, „Politische Feierkonkurrenz im Jahre 1987. Die doppelte 750-Jahr-Feier in Ost- und WestBerlin", Revue d'Allemagne et des pays de langue allemande [Online], 49-1 | 2017, Online erschienen am: 16 Juni 2018, abgerufen am 03 Juni 2022. URL: http://journals.openedition.org/allemagne/523 ; DOI: https://doi.org/10.4000/allemagne.523 


\section{Politische Feierkonkurrenz im Jahre 1987. Die doppelte 750-Jahr-Feier in Ost- und West-Berlin}

- Krijn Thijs*

Berlin gehörte lange Zeit zu den umstrittensten Städten der Welt. Das hängt nicht zuletzt mit der Geschichte des 20. Jahrhunderts zusammen, die sich weit über Deutschland hinaus als ein Ringen dreier politischen Gesellschaftsordnungen erzählen lässt: des Faschismus, des Kommunismus und der freiheitlichen Demokratie ${ }^{(1)}$. Jedes dieser Systeme schuf sein eigenes Berlin. Sie prägten die Stadt durch Architektur und Planung, durch die Bindung und Beeinflussung der jeweiligen Stadtbevölkerung und durch öffentliche Ereignisse und Selbstdarstellungen. Zu diesen letzteren, im politisch-kulturellen Bereich anzusiedelnden Repräsentationen zählen die Feierlichkeiten zu drei auffälligen städtischen Geburtstagen. Denn mehr oder weniger zufällig inszenierte jedes der drei genannten Systeme ein eigenes Berliner Stadtjubiläum. Die erste Geburtstagsfeier fand im Jahre 1937 statt, das 700jährige Jubiläum der damaligen nationalsozialistischen Reichshauptstadt. Folgerichtig gab es 1987 eine 750-Jahr-Feier, und zwar in doppelter Ausführung: einmal in der östlichen Hauptstadt der DDR und einmal in der ummauerten Inselstadt West-Berlin. Dreimal also feierte Berlin im vergangenen Jahrhundert Geburtstag, und jedes Mal unter anderen ideologischen Vorzeichen. Die vergleichende Erforschung dieser Feste bietet einen aufschlussreichen Einblick in die gesellschaftliche und ideologische Zeitgeschichte der Stadt. Dieser Beitrag gibt einen Überblick über die Ziele, den Verlauf und das Erbe der drei historischen Jubiläen, mit einem besonderen Interesse für ihre Interaktionen und Abgrenzungen ${ }^{(2)}$.

* Dr. Krijn Thijs ist wissenschaftlicher Mitarbeiter am Duitsland Instituut Amsterdam der Universität Amsterdam.

1 Siehe Mark Mazower, Dark Continent. Europe's twentieth century, London, 1998.

2 Siehe Krijn Thiss, Party, Pomp und Propaganda. Die Berliner Stadtjubiläen 1937 und 1987, Berlin, 2012. 


\section{Politik der Stadtjubiläen}

Die Systemkonkurrenz in Berlin kreierte eine ganz besondere Feierkonkurrenz zu den Geburtstagen der Stadt. Denn natürlich waren die Jubiläen von 1937 und 1987 mehr als nur fröhliche Volksfeste mit Berliner Bären und geschmückten Straßen. Stets begriffen die Stadtverwaltungen die Feiern als höchst politisch, und das zu Recht. Gerade im scheinbar unpolitischen Gewand einer Geburtstagsfeier ließ sich politische Orientierung anbieten: durch emotionale Bindungen, Freund-Feind-Bilder, Gemeinschaftserfahrungen und die Verortung in der Geschichte ${ }^{(3)}$. Die Berlin-Inszenierungen konnten kaum unterschiedlicher ausfallen als in der Hauptstadt des Dritten Reiches, der DDR-Metropole und dem West-Berliner Biotop - und das machte etwaige Ähnlichkeiten immer wieder so brisant.

Bis 1937 hatte Berlin keine Tradition von Gründungsfeiern oder Jubiläen. Die Idee wurde erstmals in den zwanziger Jahren diskutiert. Damals richteten viele deutsche Städte Ortsjubiläen aus, darunter 1929 Brandenburg mit seiner 1000-Jahr-Feier. 1928 wurde die Berliner Stadtverwaltung gefragt: Könne nicht auch Berlin eine Geburtstagsfeier machen? Der Archivar bestätigte „1230 oder 1231“ als Gründungsjahr von Berlin - „höchstwahrscheinlich“, wie er schrieb, und irrtümlich, wie man heute weiß, denn die Stadt ist etwas älter ${ }^{(4)}$. Damals aber lehnte Oberbürgermeister Gustav Böß die Idee einer 700-Jahr-Feier ab. Ein festes Gründungsdatum sei nicht überliefert, und darüber hinaus meinte Böß, dass „die gegenwärtigen Zeitverhältnisse für die Abhaltung prunkvoller Feste doch wohl nicht geeignet“ seien. „Politische Gründe kämen für eine derartige Feier ebenfalls nicht in Frage “(5). In der krisengeschüttelten Metropole der Weimarer Republik war eine teuere Selbstfeier nicht opportun.

Das sah acht Jahre später ganz anders aus. Der nationalsozialistische Kommunalpolitiker Julius Lippert festigte Ende 1936 endlich seine lang ersehnte Herrschaft über die Stadtverwaltung, die er seit 1933 gesäubert hatte. Als neuer „Stadtpräsident und Oberbürgermeister“ hatte Lippert viele Gründe für ein prunkvolles Fest, mit dem er sich und sein neues Amt inszenieren konnte. So reaktivierte Lippert die alte Idee einer 700-JahrFeier und legte sie für den Sommer 1937 fest. Als Begründung diente nun die urkundliche Ersterwähnung von Cölln. Damit stifteten die Berliner Nazis eine bis heute wahrende Tradition: folgerichtig wurde 1987 und, viel bescheidener, 2012 erneut gefeiert ${ }^{(6)}$.

\section{Die 700-Jahrfeier der Reichshauptstadt 1937}

Die ersten, die den Geburtstag der Stadt überhaupt begingen, waren also die Nationalsozialisten. Ihre 700-Jahr-Feier 1937 war eine lokale Veranstaltung. Das Stadtfest sollte die Gemeinschaft und Heimatliebe der Berliner festigen und die Stadt in das

3 Siehe Adelheid von SAldern (Hg.), Inszenierter Stolz. Stadtrepräsentationen in drei deutschen Gesellschaften (1935-1975), Stuttgart, 2005.

4 Landesarchiv Berlin (LAB), A Rep 021-2, 49: Ernst Kaeber an das Nachrichtenamt, 19.1.1928. Vgl. Norbert Meier, Berlin im Mittelalter. Berlin/Cölln unter den Akaniern, Berlin, 2012; Wolfgang FRITze, Gründungsstadt Berlin. Die Anfänge von Berlin-Cölln als Forschungssproblem, Berlin, 2000.

5 LAB, A-Rep 021-2, Aktennotiz, 6.2.1928.

6 Dazu Krijn Thijs, „An der Marienkirche. Die Berlinjubiläen von 1937 und 2012. Ein Vergleich“, Berliner Museumsjournal, 4 (2012), S. $18 \mathrm{f}$. 
Dritte Reich eingliedern. Das Programm dauerte eine Woche im August und umfasste neben dem Festzug auch ein Festspiel im Olympiastadion, eine Freiluftausstellung und einen Blumenkorso. Das alles wurde zwar mit viel nationalsozialistischem Pathos aufgeladen, bewegte sich zugleich aber innerhalb des für Stadtfeste damals üblichen Rahmens. Kein Albert Speer und keine „Germania“-Planungen - Berlins 700-JahrFeier war nicht mehr und nicht weniger als ein Heimatfest. Stadtpräsident Lippert ließ sich feiern und ordnete seine Stadt und ihre Bevölkerung ostentativ in das Dritte Reich ein. „Ich bin überzeugt“, verkündete er, „daß alle Berliner Volksgenossen heute stolz auf ihre Heimatstadt sind und mich im Zeichen der 700-Jahr-Feier darin unterstützen werden, eine Volksgemeinschaft zu schaffen, die in vorderster Reihe in dem Kampf um Deutschlands Größe steht “(7). Und in der Tat beteiligten sich die Berliner rege am Jubiläumsfest: Sie säumten in großer Zahl den Festzug, schmückten ihre Fenster und nahmen am Blumenkorso teil.

Die NSDAP-Parteigrößen zeigten wenig Interesse an dem lokalen Ereignis. Hitler blieb dem Jubiläum fern, und der populäre Berliner Gauleiter Joseph Goebbels nahm als Ehrengast nur einen Tag daran teil. In seiner Festrede blickte er auf die „Kampfzeit" nach 1926 zurück, grenzte das Dritte Reich von der verhassten Zeit der Republik ab und stellte fest: „Es ist uns gelungen, in knapp zehn Jahren aus dieser nach Moskau einst rötesten Stadt der Welt wieder eine wahrhaft deutsche Stadt zu machen“(8). Anschließend amüsierte er sich beim großen Festumzug. In seinen Tagebüchern spottete er aber. Lippert habe „gar kein Format für Berlin“, und seine 700-Jahr-Feier sei „ein wahrer Witz“. Goebbels weiter: „Berlin hat sich zum 700 Jahrfest geschmückt. Sehr pompös, aber wenig geschmackvoll. Typisch Lippert“(9).

Der historische Festzug war bei Stadtjubiläen der traditionelle Höhepunkt, und so war es auch 1937 in Berlin. Das Geburtstagsfest wurde am Sonntag, dem 15. August, mit einer historischen Parade eröffnet, an der etwa 4.300 Darsteller, 70 Wagen und 10 Musikkapellen teilnahmen. Thema der 130 Bildfolgen war laut Programmheft „das Werden und Wachsen unserer Heimat, der Stadt, die aus einer kleinen Stadtgründung auf ostdeutschem Kolonialboden zur Hauptstadt des Dritten Reiches geworden ist “(10). Der Festzug wurde vom Organisationsausschuss inhaltlich und politisch sorgfältig vorbereitet und veranschaulichte in seinem ersten Teil die Stadtgeschichte in szenischen Bildern. Im zweiten Teil stellten sich die Berliner Bezirke dar, die erst 17 Jahre zuvor eingemeindet worden waren und teilweise über eine ausgeprägte eigene Identität verfügten. In einem abschließenden, etwas kürzeren Teil präsentierte sich das nationalsozialistische Berlin der Gegenwart: Neben bunten Wagen der Industrie marschierten Hitlerjugend und Soldaten. Für die Größe der Parade spielte die traditionelle deutsche Städtekonkurrenz eine wichtige Rolle, in diesem Fall die Rivalität mit München. Dort war im Juli 1937 mit einem beeindruckenden Festumzug der Tag der deutschen Kunst

7 Lippert, zitiert nach Berliner Morgenpost, 15.8.1938. Vgl. Christoph KreuzmüLler und Michael Wild (Hg.), Berlin 1933-1945, Berlin, 2013; Gianluca Fal Anga, Berlin 1937. Die Ruhe vor dem Sturm, München, 2007.

8 Goebbels, zitiert nach Völkischer Beobachter, 17.8.1937.

9 Einträge vom 13. und 14.8.1937, in: Elke FröHLICH (Hg.), Die Tagebücher von Joseph Goebbels, Bd. I/4, München, 2000, S. 263f.

10 LAB 001-2, 428: Programmheft, S. 1. 
begangen worden. Der von Lippert kontrollierte Organisationsausschuss im Roten Rathaus beschloss umgehend, dass „der Berliner Festzug dem Münchener Festzug ohne Rücksicht auf Geld - nicht nachstehen“ dürfe und dass „die Ausgestaltung des Berliner Festzuges möglichst pompös vorgenommen werden“ solle ${ }^{(11)}$. Der Zug legte schließlich eine Strecke von nicht weniger als zwanzig Kilometern zurück und zog überall in Berlin - trotz gelegentlicher Regenschauer - Tausende von Zuschauern an.

Anders als der Festzug durch die Stadt war das historische Festspiel im Olympiastadion ein Musterbeispiel nationalsozialistischer Massenchoreographie. Hier wurde die Stadtgeschichte szenisch umgesetzt, mit Trompetenfanfaren, Glockengeläuten und Scheinwerfern im dunklen Stadion. Im ausverkauften Olympiastadion verfolgten die Berliner gebannt die abwechslungsreichen Massenszenen. „Das Festspiel ist ein Spiel des Volkes für das Volk, Zehntausende spielen für Hunderttausende. Möge es alle zusammenschließen zu einer wahrhaft freudig bewegten und festlich erhobenen Gemeinschaft", wünschte sich der Intendant Hanns Niedecken-Gebhard im Programmheft $^{(12)}$. Das Festspiel hatte einen solchen Erfolg, dass zusätzlich zu den drei geplanten Aufführungen noch drei weitere angesetzt wurden.

So gehören die Jubiläen untrennbar zur politischen Geschichte Berlins dazu: Sie machten durch Festzüge und Ausstellungen die Gemeinschaft erfahrbar, den Stadtkörper gleichsam sichtbar und den Geschichtsverlauf anschaulich. Ob und inwiefern die Bevölkerung diese Sinn- und Identifikationsangebote auch tatsächlich annahm, steht auf einem anderen Blatt. Diese Frage nach Dissens und Protest ist für das doch ziemlich kleine und kurze Stadtjubiläum von 1937 schwieriger zu beantworten als für 1987.

\section{Die Ost-Berliner 750-Jahr-Feier 1987}

Ein halbes Jahrhundert später war Berlin eine geteilte Stadt. Und 1987 war ein Ende der Teilung noch nicht absehbar (rückblickend vielleicht schon, zeitgenössisch aber nicht). So standen sich beide Stadthälften zum Jubiläum in voller Ausprägung gegenüber.

Ost-Berlin, das als Sowjetsektor lange Zeit im Schatten des legendenumwobenen West-Berlin gestanden und 1961 mit dem Bau der Mauer die Spaltung der Stadt besiegelt hatte, erlebte seit den 1970er Jahren so etwas wie einen Aufschwung. Die zunehmende internationale Anerkennung der DDR, und insbesondere das Viermächteabkommen über Berlin im Jahr 1971, hatten auch das Ansehen Ost-Berlins gesteigert. Es richtete sich in der Ära Honecker in seiner Rolle als „Hauptstadt der DDR“ ein, die die Westmächte indes nicht anerkannten. Die rechtlich umstrittene Situation führte zu einer nahezu obsessiven Empfindlichkeit in Statusfragen. So führte OstBerlin seine Funktion stets im Namen mit: „Berlin, Hauptstadt der DDR“.

Im Osten kam das anstehende Stadtjubiläum um 1980 auf die Agenda und wurde sofort als Staatsereignis eingestuft. Politbüromitglied Kurt Hager erklärte 1981, das Jubiläum könne erstens „einen wesentlichen Beitrag bei der Festigung des sozialistischen Heimatund Nationalbewusstseins der Bürger der DDR leisten“, zweitens „das internationale Ansehen Berlins als sozialistische Metropole stärken“ und schließlich „imperialistische

11 Ebd., S. 431: Vermerk zum Festzug, 17.7.1937.

12 Hanns Niedecken-Gebhard, „Zum Ausklang“, in: 700-Jahr-Feier der Reichshauptstadt. Folge der Feier vom 14. bis 22. August 1937, Berlin, 1937. 
Konzeptionen von einer, Gesamtberliner Geschichte‘ bzw. vom ,Offenhalten der deutschen Frage wirksam zerschlagen helfen“(13). Anders als im Dritten Reich gewann das Fest in der DDR direkt staatspolitischen Rang. Damit war eines von vornherein klar: Man würde 1987 auf gar keinen Fall mit West-Berlin zusammen feiern.

In den folgenden Jahren geriet die DDR aber in eine Phase der Stagnation, in erster Linie wirtschaftlich, aber auch politisch und sozial. Der notwendige Generationswechsel kam nicht zustande, die Zahl der Ausreiseanträge stieg, und es entwickelten sich, vor allem in der Hauptstadt und unter dem Dach der Kirche, oppositionelle und ,unabhängige‘ Gruppen. Ausgerechnet im Realsozialismus fehlten überzeugende Konzepte für die Zukunft. Dennoch feierte Honecker 1987 mit seinem offiziellen Besuch in Bonn seinen größten diplomatischen Triumph - der DDR-Chef galt nun als Staatsmann und manchen sogar als Friedensbotschafter.

Unterdessen hatte die geplante 750-Jahr-Feier gewaltige Dimensionen angenommen, angetrieben von der direkten Legitimationskonkurrenz zu West-Berlin. Der Staats- und Parteichef stand selbst dem Vorbereitungskomitee vor, das im Februar 1985 gegründet wurde und dem, wie Honecker betonte, nicht weniger als „169 Persönlichkeiten aus allen Bereichen des gesellschaftlichen Lebens unserer Republik“ angehörten $^{(14)}$. Die eigentlichen Weichenstellungen wurden hinter den Kulissen im Zentralkomitee der SED vorgenommen und vom Politbüro bestätigt, während die Berliner Stadtverwaltung eher ausführende Aufgaben übernahm. Der Rang der Feier spiegelte sich besonders im Wohnungsbau und in der Pflege des Stadtbildes. Nach Jahrzehnten modernistischer Stadtplanung leistete sich die DDR nun einige prominente Rekonstruktionsprojekte. Am Platz der Akademie (dem heutigen Gendarmenmarkt), der lange eine imposante Ruinenlandschaft gewesen war, wurden zwischen 1978 und 1987 das Schauspielhaus, der Französische Dom und der Deutsche Dom wieder aufgebaut. Ebenso überraschend war die Wiedererstehung des Nikolaiviertels, Berlins Geburtsstätte. Sie stattete Ost-Berlin mit einer historischen Kulisse aus, die im Realsozialismus durchaus spektakulär war ${ }^{(15)}$. Und sie unterstrich zudem, dass die Wurzeln der Stadt diesseits der Mauer lagen und nicht drüben, in West-Berlin. Böse Zungen witzelten aber über das Nikolaiviertel, das wie ein abgeschlossenes Sonderviertel mit guter Versorgung und hübscher Kulisse ein scharfes Kontrast zur grauen DDR-Realität abgab („Honni’s Disneyworld“).

Im Wettrennen mit West-Berlin um die größte 750-Jahr-Feier hatte sich das Programm in Ost-Berlin auf das ganze Jahr ausgestreckt. Es gab internationale Konferenzen, Jahrmärkte, Bücher, Ausstellungen, gute Versorgung, Rockkonzerte und Volksfeste ${ }^{(16)}$. Der Geburtstag selbst wurde mit einem Staatsakt im Palast der Republik gefeiert. Hier malte Honecker stolze Wunschbilder: „Mit dem heutigen Staatsakt finden die Feierlichkeiten zum 750-jährigen Jubiläum Berlins ihren Höhepunkt. [...] Während der zurückliegenden Monate war besonders eindrucksvoll zu spüren, wie kraftvoll der Puls des Lebens in unserem Lande schlägt. Berlin, die Stadt des Friedens, erwies sich als weltoffener

13 Kurt Hager an Konrad Naumann, 9.7.1981, in: Bundesarchiv Berlin SAPMO (BA), DY30/vorl SED 38791.

14750 Jahre Berlin. Konstituierung des Komitees der DDR, Berlin, 1985.

15 Florian Urban, Berlin/DDR, neohistorisch. Geschichte aus Fertigteilen, Berlin, 2007.

16 Siehe 750 Jahre Berlin. Das Buch zum Fest, Berlin, 1986. 
Ort der Begegnung, des Dialogs und der Zusammenarbeit, als Anziehungspunkt für namhafte Künstler und Ensembles der internationalen Kultur“(17).

Unbestrittener Höhepunkt der 750-Jahr-Feier war der große historische Festzug, der am 4. Juli 1987 bei strahlendem Sonnenschein durch die Magistralen der Hauptstadt rollte. Im Vergleich zu 1937 war die Strecke von Unter den Linden bis zur Karl-Marx-Allee zwar kurz, die Zahl der Mitwirkenden mit 40.000 Menschen dafür aber fast zehn Mal so hoch. Der Zug umfasste 291 Bildfolgen und dauerte volle fünf Stunden. Es kostete die kriselnde DDR aber erhebliche Energien, um dieses Wagnis ,störungsfrei' über die Bühne zu bekommen. Der Platz um die Haupttribüne an der Karl-Liebknecht-Straße war weiträumig abgeriegelt und mit ausgewähltem Publikum besetzt. Die Beamten der Staatssicherheit befanden sich im Großeinsatz ${ }^{(18)}$. Doch der Tag wurde ein großer Erfolg. Der Festzug überraschte Freund und Feind mit seiner ideenreichen Ausstattung. Ganz anders als die üblichen sozialistischen Aufmärsche verströmte er Lockerheit und echte Volksfeststimmung - darüber waren sich Informanten des ostdeutschen Geheimdienstes und westdeutsche Journalisten einig.

Inhaltlich orientierte sich der Zug an den Thesen 750 Jahre Berlin, einer parteioffiziellen Kurzdarstellung der Berliner Geschichte, die als Leitfaden für alle historisch ausgerichteten Veranstaltungen des Jubiläums diente ${ }^{(19)}$. Damit sorgte Ost-Berlin für ein einheitliches Geschichtsbild, das auf Klassenkämpfe und den gesetzmäßigen Fortschritt zum Sozialismus abhob. Dabei nahm man die Zeit nach 1949, beschränkt auf Ost-Berlin, mindestens ebenso wichtig wie die 700-jährige Geschichte zuvor: Die DDR galt offiziell als Höhepunkt und Erfüllung der Berliner Stadtgeschichte.

Der Festzug sparte die sensiblen Kapitel der Ost-Berliner Geschichte nicht aus. Das galt beispielsweise für die ,Befreiung' 1945 durch die Rote Armee, die viele ältere Berliner mit schrecklichen Erfahrungen verbanden. Sogar die Berliner Mauer kam im Festzug vor. In der Bildfolge 13. August - wir schützen zuverlässig die Grenzen der Republik und den Frieden rollte das zugemauerte und von Veteranen überwachte Brandenburger Tor im Zug mit. Ein Thema der Nachkriegsgeschichte fehlte in Ost-Berlin jedoch völlig, und das war West-Berlin. Weder in den Geschichtsdarstellungen noch in den Festveranstaltungen wurde auf die Entwicklung im Westteil eingegangen - wie selbstverständlich verengte sich die Berliner Stadtgeschichte ab 1949, dem Gründungsjahr der DDR, auf die östliche Hälfte.

Alles in allem war es durchaus spektakulär, was Ost-Berlin zum Berlin-Geburtstag auf die Beine brachte. Doch die mühsam aufrechterhaltene Fassade zeigte (vor allem im Rückblick) bereits viele Risse. Michael Gorbatschow kam nach Berlin und wurde besonders laut bejubelt, während die offiziellen Beziehungen zur SED-Führung bereits sehr abgekühlt waren. Im April degradierte Kurt Hager die Perestrojka zum „Tapetenwechsel“" und Demonstranten zeigten bei den offiziellen 1. Mai-Kundgebungen dann zahlreiche Gorbatschow-Bilder ${ }^{(20)}$.

17750 Jahre Berlin. Staatsakt der DDR am 23. Oktober 1987, Berlin, 1988.

18 Siehe Das Bilderbuch vom Festumzug, Berlin, 1987; Jens Sснӧne, Stabilität und Niedergang. Ost-Berlin im Jahr 1987, Berlin, 2006.

19750 Jahre Berlin. Thesen, Berlin, 1986. Ausführlich zu den Geschichtserzählungen bei den Feiern: Krijn Thijs, Drei Geschichten, eine Stadt. Die Berliner Stadtjubiläen 1937 und 1987, Köln u.a., 2008.

20 Zu den Protestkulturen: J. Schöne, Stabilität (Anm. 18); Ilko-Sascha Kowalczuk, Endspiel. Die Revolution von 1989 in der DDR, München, 2009. 
Auch bei manchen Jubiläumsveranstaltungen wurde die Opposition sichtbar. Zu Pfingsten kam es bei West-Berliner Rockkonzerten an der Mauer zu Unruhen in Ost-Berlin - darauf komme ich noch zurück. Ende Juni fand in Berlin ein kritischer „Kirchentag von unten“ statt, wo öffentlich über Glasnost und Perestrojka debattiert wurde. Zudem setzte die alternative Szene der Staatspropaganda eigene Berlin-Bilder entgegen. So zeigten der Fotograf Harald Hauswald und der Autor Lutz Rathenow in der Umweltbibliothek erstmals ihre Fotoausstellung Ostberlin - die andere Seite einer Stadt. Ihr Projekt war auch gegen das Jubiläum gerichtet und dokumentierte Aussteiger, Alltag und Jugendkulturen der Hauptstadt und wurde über die DDR hinaus bekannt. Hauswalds Bilder wurden auch im Westen sehr bekannt, unter anderem durch die Zeitschrift GEO, die 1986 eine Sonderausgabe zu den Szenen in West- und Ost-Berlin herausbrachte und mit seinen Fotos illustrierte ${ }^{(21)}$.

Somit war der hohe politische Rang, der dem Fest staatlicherseits zuerkannt wurde, auch eine Belastung für das Jubiläum. Es blieb dadurch wenig Raum für die Betonung einer eigenständigen Berliner Identität. Zudem stieg überall im Land der Ärger auf die Hauptstadt, die für 1987 noch offensichtlicher bevorzugt wurde als ohnehin schon üblich. Über Berlin zu lästern wurde 1987 in der Region fast zu einer patriotischen Bürgerpflicht. Es gab Sprüche und Witze ohne Ende: „Für Berlin das Beste, für die Republik die Reste“; „Kann man ohne Ekel bis 1.000 zählen? Nein, man muss an den 750 vorbei“; „Das Versorgungsproblem im Sozialismus ist gelöst: Wir schaffen alles nach Berlin, und dort holt sich jeder ab, was er braucht“. Bauarbeiter aus der gesamten Republik wurden nach Berlin beordert, um die Hauptstadt herauszuputzen, während zuhause die Innenstädte weiter zerfielen. So kam es, dass in Berlin Zementlaster aus der Provinz mit Losungen wie „1026 Jahre Halle“ fuhren. Autoaufkleber und Signets wie „781 Jahre Dresden“ und „821 Jahre Leipzig“ wurden vom Staat heuchlerisch zu Zeichen sozialistischer Heimatliebe umgedeutet ${ }^{(22)}$. Nicht selten endete der Berlin-Hass in regelrechtem Vandalismus: Mehrfach wurden im Umland Berliner Autos beschädigt oder mit Farbe beschmiert („Ärsche 750“). Die entsprechenden Motive wurden hungrig von westlichen Medien aufgegriffen. So mischte sich die Berlinfeierei mit allerlei alltäglichen Themen des Spätsozialismus, und im Westen war das im Grunde nicht anders.

\section{Die West-Berliner 750-Jahr-Feier 1987}

Mitte der 1980er Jahre war West-Berlin einer der merkwürdigsten Orte Europas. Seit fast vierzig Jahren hatte die halbe Metropole nun schon überlebt, eingemauert und doch frei, eine Enklave in feindlichem Land. Durch das Berlinabkommen 1971 hatte sich ihre exponierte geografische Lage zwar inzwischen stabilisiert. Doch das warf letztlich die Frage auf: Wozu ist West-Berlin eigentlich noch da? Die Insel war vollkommen abhängig von Subventionen aus der Bundesrepublik und erlebte 1981 ein regelrechtes Krisenjahr mit Bau- und Korruptionsskandalen, Hausbesetzern und Straßenschlachten und gar drei Regierenden Bürgermeistern. Zunehmend schien Berlins neue Aufgabe darin zu liegen, innerhalb der Bundesrepublik neue alternative

21 Siehe Harald Hauswald, Lutz Rathenow, Ost-Berlin. Die andere Seite der Stadt, Berlin, 1990. Vgl. GEO Special, Nr. 6, 3.12.1986 (Themenheft Berlin).

22 Wolfgang Oschlies, „,Man nennt uns manchmal Fischkopf... Zum DDR-Regionalismus und seinen subkulturellen Reflexen“, Deutschland Archiv, 21 (1988), S. 778-786. 
Strömungen $\mathrm{zu}$ integrieren. Als faszinierender (aber inzwischen ungefährlicher) Grenzraum zog die Halbstadt nicht nur bundesdeutsche Wehrdienstverweigerer, sondern auch Studenten, Künstler und Abenteurer aus der gesamten westlichen Welt an. Sie pflegten auf ihrer Insel eine leicht überhebliche Äquidistanz sowohl zum weit entfernten Westdeutschland mit seinen spießigen Berlinbesuchern und seinem provinziellen Kanzler als auch zur ungeliebten DDR, der sie hauptsächlich in Gestalt ihrer launischen Grenzbeamten im gespenstischen Transitverkehr begegneten. In Berlin tickten die Uhren eben anders. Nationale Identität, Wiedervereinigungsgebot und Hauptstadtambitionen waren für weite Teile der jüngeren Generation Begriffe aus einer anderen Welt. Politisch regierte aber ab 1981 das konservative Berlin die Stadt, erst mit Richard von Weizsäcker, dann ab 1984 mit Eberhard Diepgen ${ }^{(23)}$.

Für die Insel war der 750. Geburtstag zunächst ein schwieriges Datum. Es gab in den 1980ern wenig Anlass für stolze Selbstinszenierungen. Die historische Altstadt lag hinter der Mauer. Zudem wurden im Abgeordnetenhaus nachdrücklich Vergleiche angestellt: „Wir wollen nicht, dass eine Feier per Dekret stattfindet, etwa wie jene, die jetzt mit militärischem Pomp in Ost-Berlin vorbereitet wird“, hieß es dort ${ }^{(24)}$. Ursprünglich hatte man auf gemeinsame Veranstaltungen mit Ost-Berlin gehofft, um die Zusammengehörigkeit zu unterstreichen. Mit einem getrennt-doppelten Jubiläum, so die Abgeordneten ganz richtig, würde sich Berlin „mehr oder weniger der Lächerlichkeit" der Weltöffentlichkeit aussetzen ${ }^{(25)}$. Doch Ende 1985 zerschlugen sich alle Hoffnungen. Ost-Berlin zog ein eigenes Staatsfest auf und blendete sein ,Vorstadtgebiet' aus. So musste West-Berlin allein die Einheit der Stadt vor Augen führen. „Das Geschichtsfest wird doppelt begangen, aber gemeinsam gesehen“, verkündete deshalb das Programmbuch, das ganz bewusst auch auf die Ost-Berliner Veranstaltungen hinwies $^{(26)}$. Als zentralen Veranstaltungsort wählte West-Berlin das Gebiet „vor den Toren der Stadt“. Die doppeldeutige Formel bezeichnete historisch die Gegend entlang der früheren Stadtgrenze zwischen Brandenburger Tor, Potsdamer Tor, Halleschem Tor usw. Hier verlief jetzt aber die Berliner Mauer, und so war die Festchoreographie vor den Toren 1987 durchaus auch politisch gemeint, wie es die Richtlinien des Senats ausdrückten: „Die Mauer kann und darf nicht die Mitte Berlins zur Peripherie machen. Der Senat wird das Jahr 1987 bewußt mit dem Blick auf den Reichstag und auf die Mauer - aber auch über sie hinweg - begehen“ “(27).

In den 1980er Jahren war West-Berlin ohnehin dabei, diese lange vernachlässigte Mitte der alten Reichshauptstadt wiederzuentdecken ${ }^{(28)} .1987$ gab es hier eine

23 Siehe Wilfried Rotт, Die Insel. Eine Geschichte West-Berlins 1948-1990, München, 2009. Spezifisch zu den späten 1980er Jahren: Krijn Thijs, „Entfernter Erfahrungsraum. Überlegungen zu West-Berlin und 1989“, Eurostudia, 7/1F. (2011), S. 29-46; Stefanie EISEnhuth, West-Berlin und der Umbruch in der DDR. Grenzübergreifende Wahrnehmungen und Verhandlungen 1989, Berlin, 2012.

Abgeordnetenhaus von Berlin, Plenardebatte 9/28, 23.9.1982, S. 1737B (Biewald, CDU).

Abgeordnetenhaus von Berlin, Plenardebatte 9/41, 10.3.1983, S. 2445A (Kollat, SPD).

Ulrich Eckнавdт (Hg.), 750 Jahre Berlin. Stadt der Gegenwart, Berlin, 1987 (Klappentext).

Abgeordnetenhaus von Berlin, Drucksache 9/2292, 30.11.1984, S. 3.

Siehe Krijn Thiss, „West-Berliner Visionen für eine neue Mitte. Die Internationale Bauausstellung, der ,Zentrale Bereich ' und die ,Geschichtslandschaft' an der Mauer (1981-1985)“, Zeithistorische Forschungen, 11/2 (2014), S. 235-261. 
Reihe von Renovierungsprojekten, beispielsweise den Hamburger Bahnhof, den Martin-Gropius-Bau und die Kongresshalle. Im geplanten Filmmuseum im Hotel Esplanade gab es eine erste Ausstellung; im Kino lief Wim Wenders Der Himmel über Berlin, eine Hommage an den ehemaligen Potsdamer Platz. Am Kulturforum wurde die Philharmonie um den Kammermusiksaal erweitert und eine Probestrecke der futuristischen Magnetbahn aufgebaut. Auch die Gründung des Deutschen Historischen Museums passte zu dieser Strategie, die Mitte wiederzubeleben. Seinen Sitz sollte das neue Museum gegenüber dem Reichstag haben, und Bundeskanzler Helmut Kohl enthüllte zum Geburtstag der Stadt den Grundstein.

Hier in der leeren Mitte fand auch die große Jubiläumsausstellung Berlin, Berlin statt, im Gropiusbau, wo West-Berlin seine stolze Metropolengeschichte inszenierte. Die Besucher sollten die dynamische Geschichte der Großstadt sinnlich erfahren. Beispielhaft dafür stand die Inszenierung im großen Lichthof, die unter dem Titel Die schnellste Stadt der Welt die verlorene Metropole der Weimarer Republik vor Augen führte. Zum Geschichtsbild gehörten auch die Schattenseiten der Berliner Geschichte, die an diesem Ort sowieso präsent waren. Der Haupteingang des Martin-GropiusBaus wurde von der Berliner Mauer versperrt. Nebenan lag das Brachgelände des ehemaligen Reichssicherheitshauptamtes, Hauptsitz der SS. Auf Betreiben von Bürgerinitiativen wurde die Geschichte dieses ,Gestapo-Geländes` 1987 erstmals in einer Begleitausstellung aufgearbeitet - der Ursprung der heutigen Dauerausstellung Topographie der Terrors. So präsentierte West-Berlin 1987 ein durchaus nachdenkliches Ausstellungsprogramm.

So wie sich Gorbatschow und die führenden Köpfe des Warschauer Paktes im Osten zeigten, so kamen die westlichen Alliierten zum Geburtstag nach West-Berlin. François Mitterrand, die britische Queen Elizabeth II. und schließlich der amerikanische Präsident Ronald Reagan besuchten ihr exponiertes Schutzgebiet. Die amerikanische Geburtstagsparty fand am 12. Juni in einem Hangar am Flughafen Tempelhof statt. Anschließend hielt Ronald Reagan seine berühmte Rede vor dem Brandenburger Tor ${ }^{(29)}$.

Mit solchen Bauprojekten, Ausstellungen und Staatsbesuchen hatte West-Berlin seine Jubiläumsfeier weitgehend beisammen. Die zentralen Festveranstaltungen allerdings verlangten vom Organisationsbüro B750 besondere Aufmerksamkeit und Kreativität. Denn die nationalsozialistische 700-Jahr-Feier hatte eine Reihe traditioneller Festformen diskreditiert. Dies galt an erster Stelle für den Festzug. Bereits in den ersten Ideenskizzen wurde festgehalten, dass „alle Peinlichkeiten mit historisierenden Aufzügen und einer dazu nötigen Massenregie“ zu vermeiden seien ${ }^{(30)}$. Auch große historische Aufführungen im Olympiastadion waren nicht wiederholbar. Volksfeste sollte es 1987 aber unbedingt auch geben, hier musste West-Berlin sich also erfinderisch zeigen. Das gelang zuallererst mit dem „Wasserkorso“, einem Festzug auf dem Wasser, zu dem historische Schiffe aus ganz Westeuropa eingeladen wurden. So konnte man den gefürchteten Gleichschritt vermeiden und sich zugleich als weltoffen präsentieren. „Kein Jubiläum ohne Festzug!“, erklärten die Veranstalter. „Doch diesmal muss nicht

29 Zu den Hintergründen: Helmut von Trotnow, Florian WeIss (Hg.), Tear down this Wall. US-Präsident Ronald Reagan vor dem Brandenburger Tor, 12. Juni 1987, Berlin, 2007.

30 LAB, B Rep 150, 54: Ideenskizze, 19.9.1983. 
marschiert werden, denn der Umzug findet auf dem Wasser statt, auf den Kanälen, den Havelseen und natürlich auf der Spree, die unbeeindruckt von Grenzen die Stadt wie vor 750 Jahren in ihrem alten Bett durchfließt“(31).

Ein weiterer Höhepunkt des Jubiläumsfestes wurden die multimedialen Inszenierungen an der Siegessäule. Die SternStunden präsentierten im August eine Theaterund Musik-Revue zur Geschichte Berlins. Auch das war als demokratische Variante des Stadtfestes gedacht. Statt des Olympiastadions wählte West-Berlin einen öffentlichen Park, den Tiergarten, und organisierte hier eine Show nicht auf der Basis von Massenchoreografien, sondern mit Einzelauftritten und technischen Projektionen. Rückblickend erklärte Kultursenator Volker Hassemer emphatisch, dass die SternStunden „die 700-Jahr-Feier aufgehoben“ hätten. Die Siegessäule sei in eine „Friedenssäule" umgewandelt worden ${ }^{(32)}$.

Dennoch gab es auch in West-Berlin viel Kritik und am Ende auch Gewalt. Im Laufe des Feierjahres wurde der nicht ablassende Strom an Festveranstaltungen, Reden, Empfängen und Ausstellungen von wachsendem Spott und Zynismus begleitet, zumal es in Kreuzberg im Mai und Juni schwere Krawalle gegeben hatte, die durchaus von der dauernden politischen Selbstdarstellung beflügelt wurden. In der Hektik verstiegen sich Senatoren gar zu der Aussage, dass die Randalierer keine Berliner gewesen seien, sondern „Anti-Berliner“, was in Kreuzberg fortan als stolze Selbstbezeichnung aufgenommen wurde. Im Oktober nahmen die „Anti-Berliner“ mit einer satirischkarnevalistischen B750-Antiparade den Ku'damm in Besitz ${ }^{(33)}$. Parolen wie „750 Jahre - es reicht“ zierten Wände und Fassaden. Darüber hinaus erregten einige avantgardistische Kunstprojekte im öffentlichen Raum den Unmut der Stadtbevölkerung, was West-Berlins liberal-tolerantes Selbstbild erheblich irritierte. So überwog im Herbst die Ernüchterung: Das Jubiläum war ein wenig zu groß geraten und hatte nicht, wie erhofft, zu klaren Fortschritten im innerstädtischen Verhältnis geführt. Oder doch?

\section{Interaktionen, Klopfzeichen und Echos}

Diese Annäherung an die Berliner Jubiläen zeigt, dass sich Stadtgemeinschaften in der Herausbildung und Schärfung ihrer Identität natürlich aufeinander beziehen und voneinander abgrenzen. Prominent war jedes Mal die klassische Städtekonkurrenz, die Berlin im Sommer 1937 etwa veranlasste, einen pompöseren Festzug aufzuziehen als München kurz vorher. In der DDR spielten konkurrierende Städteidentitäten beim Wut auf die bevorzugte Hauptstadt eine große Rolle: Zumindest in Bezug auf das Lebensalter konnten Dresden („781 Jahre“), Leipzig („821 Jahre“) oder Halle („1026 Jahre“) die mächtige Hauptstadt hinter sich lassen. Noch dazu kam 1987 die innerstädtische Ost-West-Konkurrenz um das wahre Berlin, die natürlich als Sonderfall durch den Kalten Krieg und die Stadtspaltung ausgelöst wurde.

31 LAB, B Rep 150, 54: Pressematerialien, 3.9.1984.

32 Torsten Mass (Hg.), 750 Jahre Berlin. Sternstunden. Eine Dokumentation, Berlin, 1987, S. 3.

33 Siehe Cornelia KüHN, „Trachtenumzug, Skulpturenboulevard oder B-750 Parade? Aushandlungsprozesse um die politische Repräsentation und ästhetische Gestaltung der 750-Jahr-Feier in West-Berlin“, in: Kaspar MaAse, Christoph Bareither, Brigitte Frizzoni, Mirjam Nast (Hg.), Macher - Medien - Publika. Beiträge der europäischen Ethnologie zu Geschmack und Vergnügen, Würzburg, 2014. 
Aber nicht nur solche,synchronen' Wettbewerbe prägten die verschiedenen politisch beladenen Städteidentitäten im 20. Jahrhundert. Das umstrittene Berlin prägte sich gerade auch in der, diachronen' und besonders taktschnellen Systemfolge heraus und bezog somit sein Profil auch durch dauernde historische Abgrenzungsversuche. Die Berliner Geburtstagsfeiern zeugen davon. West-Berlin betrieb mit seinem neuerfundenen „Wasserkorso“ und mit der Vermeidung des Olympiastadions als Repräsentationsbühne regelrechte Vergangenheitsbewältigung im Fest. Der Senat und das Vorbereitungsbüro setzten sich mit der braunen 700-Jahr-Feier explizit auseinander und grenzten sich von ihr ab. West-Berlin habe bewiesen, so lautete ihre Bilanz, „dass auch eine offene, demokratische Gesellschaft den großen Raum kulturell in Besitz nehmen kann und ihn nicht immer der Erinnerung an gleichgeschaltete Kolonnen einer unseligen Vergangenheit überantworten muß“(34). Solcherart Abgrenzung produzierte demokratische Legitimität.

Im Osten verliefen die diachronen Verbindungen entlang ganz anderer Linien, denn die SED zog es vor, die Existenz des nationalsozialistischen Vorgängerfestes ganz und gar zu verschweigen. Die Parteispitze selbst hatte den Hinweis darauf aus dem Manuskript der Thesen 750 Jahre Berlin herausgestrichen, und auch sonst war darüber in den DDR-Medien nichts Genaues zu erfahren. Somit musste sich die Parteiführung auch nicht für den eigenen Festzug rechtfertigen. Weil das Thema tabu war, zirkulierte es nun als verbotenes Wissen in Oppositionskreisen. Die illegale Zeitschrift grenzfall nannte das Jubiläum eine Erfindung von Joseph Goebbels. „So wird nun in beiden Teilen Berlins der 50. Jahrestag der 700 Jahrfeier begangen, irgendwelche Ähnlichkeiten beider bzw. der drei Feiern können wir leider nicht kommentieren, da wir über den Ablauf der 700 Jahrfeier zu wenig wissen“ “(35). Diese Äußerung weist einmal mehr auf die Querverbindungen zwischen den Berliner Stadtjubiläen von 1937 und 1987 hin. Ost und West bezogen sich explizit oder implizit auf das Vorgängerjubiläum und stärker noch aufeinander, indem sie miteinander konkurrierten, Ideen übernahmen, aber sich auch ostentativ voneinander abgrenzten. Ganz besonders deutlich wurde dies im Juli 1987. West-Berlin hatte, die nationalsozialistische Feier vor Augen, einen neuerlichen Festzug abgelehnt und nach langem Hin und Her den „Wasserkorso“ erfunden. Ost-Berlin fand diese Idee gut, wollte sich die Show nicht stehlen lassen und veranstaltete ebenfalls ein „Wasserfest“, überdies am selben Tag. Am 25. und 26. Juli feierten die beiden Stadthälften denselben Geburtstag auf demselben Berliner Fluss.

So sorgfältig aber die Jubiläumsfeiern geplant und vorbereitet waren, es gab 1987 doch viele unerwartete und spontane Ereignisse. Als besonders unberechenbar erwies sich der direkte Austausch zwischen Ost- und West-Berlin. Immer wieder kam es, auf den verschiedensten Kommunikationswegen, zu solchen Interaktionen; sie wurden oft als „Klopfzeichen an der Mauer“ bezeichnet. Natürlich ging die Initiative dazu häufiger von West-Berlin aus, das zum Geburtstag ja stets das Gemeinsame betonen wollte. Aber auch aus Ost-Berlin kamen Signale, vom Staat wie auch von der Bevölkerung.

Beispielhaft dafür stehen die bereits erwähnten Rockkonzerte am Reichstag samt „Mauerschlacht“ in Ost-Berlin ${ }^{(36)}$. Konzerte an der Mauer hatte es öfter gegeben,

Th. MAss, 750 Jahre Berlin (Anm. 32), S. 3.

35 Grenzfall, 6/87.

36 Für das Folgende: J. Schöne, Stabilität (Anm. 18) sowie I.-S. Kowalczuk, Endspiel (Anm. 20). 
und die West-Berliner Veranstalter sorgten ganz bewusst für die Beschallung von Ost-Berlin. So auch am Pfingstwochenende im Juni 1987, als das dreitägige Concert for Berlin stattfand, mit u.a. David Bowie, der New Model Army, Neil Young, den Eurythmics und Genesis. Doch diesmal waren die Auswirkungen verblüffend. Während sich etwa 60.000 zahlende West-Berliner entspannt auf ein Open-Air-Konzert freuten, stieg drüben Unter den Linden die Spannung. Die DDR-Jugend war bestens über das Festival informiert, und so versammelten sich am Samstagabend einige hundert Fans so nahe am Brandenburger Tor wie möglich, um David Bowie zu hören. Der Rockstar begrüßte sogar auf Deutsch seine Freunde „auf der anderen Seite der Mauer“. Die Volkspolizei war darauf nicht vorbereitet und drängte die Menge zurück. Empört reagierte diese mit Sprechchören.

Das sprach sich herum, und am nächsten Sonntag war die Ost-Berliner Fanschar auf über 2.000 Menschen angewachsen. Der DDR-Sicherheitsapparat mischte nun Zivilbeamte unter die Fans, um die „Rädelsführer“ ausfindig zu machen und die Menge zu zügeln. So richtig gelang dies nicht. Beim Auftritt der Eurythmics kam es zu schweren Auseinandersetzungen. Die Rockfans riefen „Wir wollen Gorbatschow!“ und dann sogar „Die Mauer muss weg!“.

Am folgenden Montagabend fanden sich noch einmal deutlich mehr Menschen ein. Das Brandenburger Tor war jetzt allerdings im Osten so weiträumig abgesperrt, dass das Konzert der Gruppe Genesis hier kaum noch zu hören war. Nun eskalierte die Situation. Die Jugendlichen riefen „Bullen raus!“, „Die Mauer muss weg!“, aber auch „Kreuzberg ist überall!“ und „Kreuzberger Nächte sind lang“ - eine mit einem populären Schlagertitel zum Ausdruck gebrachte Anspielung auf die Situation im anderen Stadtteil, wo es im Mai, wie die Ost-Berliner Szene sehr wohl wusste, schwere Krawalle gegeben hatte. Nun flogen Flaschen und Gummiknüppel, die Staatsorgane schritten mit brutaler Gewalt ein und nahmen etwa 120 Menschen fest. Eine solche Straßenschlacht zwischen Volkspolizei und Jugendlichen war in der DDR völlig ungewöhnlich. Die Westmedien berichteten ausführlich, nicht zuletzt weil sich verschiedene Westreporter, begierig auf diese Story, im Ost-Berliner Publikum befunden hatten und ebenfalls von den Stasimitarbeitern schikaniert worden waren. West-Berliner Fernsehen war fast live dabei, und so bekam Deutschland zur 750-Jahr-Feier auch das hässliche Gesicht Ost-Berlins zu sehen.

Die Ost-Berliner „Mauerschlacht“ zum West-Berliner Rockkonzert war noch in aller Munde, als einige Tage später Ronald Reagan anreiste ${ }^{(37)}$. Seine große Rede am 12. Juni gehörte zu den sorgfältig geplanten und inszenierten Klopfzeichen an der Mauer. Vor allem die Wahl des Ortes, die telegene Kulisse mit dem Blick auf OstBerlin, erwies sich als bestens gewählt. Diesmal war der westliche Bereich vor dem Brandenburger Tor weiträumig abgesperrt, denn es war schon tagelang unruhig in West-Berlin. Demonstrationen fanden in der City West statt, die Autonomen wurden mit Straßensperren in Kreuzberg abgeschnitten, und vor der Bühne hatte man ein ausgewähltes Publikum versammelt, darunter viele Amerikaner und auch sogenannte „Stabilisatoren“, die Störer sofort einkapseln konnten. (Die taz verglich hämisch die „Stabis“ hier mit den „Stasis“ drüben.) Nur vom Erzfeind, der doch direkt im Rücken 
des US-Präsidenten an der Mauer stand, schien man keine bösen Überraschungen zu erwarten. Die DDR-Organe verfolgten das Treiben vor ,ihrem' Brandenburger Tor mit Argusaugen. Wie erwartet richtete sich Reagan in seiner Ansprache mehrfach an die Ost-Berliner hinter ihm: „Es gibt nur ein Berlin“, sagte er auf Deutsch. Und über OstBerlin hinaus adressierte er seinen berühmten Appell an Moskau: „Mr. Gorbachev, tear down this wall“.

Reagans Besuch rief in West-Berlin neben Zustimmung auch viel Kritik hervor, denn der amerikanische Präsident galt als Kalter Krieger. So konnte es dazu kommen, dass Demonstranten beiderseits der Mauer die herrschende Ordnung mit „Gorbi, Gorbi“Rufen herausforderten - auch wenn ihre Situation in Ost- und West-Berlin unterschiedlicher kaum sein konnte. Die Abfolge von Demonstration, Konfrontation und Eskalation entwickelte sich beim Anti-Reagan-Protest am 11. und 12. Juni in West-Berlin nach bekanntem Muster. Im Übrigen gab es auch hier den Echoeffekt: Demonstranten, die am 11. Juni stundenlang vor dem Kaufhaus des Westens von Einsatzkräften eingekesselt waren, riefen in Richtung der Polizeikette: „Die Mauer muss weg!“

\section{Fazit}

So endete die Feierkonkurrenz von 1987 in Erschöpfung. Blickt man heute auf die Ereignisse zurück, so liegen die Bezüge zum großen Jahr 1989 auf der Hand. Gorbatschows Name war in aller Munde, und auf vielen Kommunikationskanälen hatten sich Ost- und West-Berlin neu aufeinander bezogen, sowohl diplomatisch kontrolliert als auch hemmungslos und subversiv. Im Osten versuchte die Staatssicherheit nach der gewissen Lockerheit und den oppositionellen Erfolgen des Feierjahres die Uhren wieder zurückzudrehen. Sie drang im November in die Berliner Zions-Kirche ein, wo die unabhängige Umweltbibliothek untergebracht war. Die Aktion misslang, weil Westmedien rasch zur Stelle waren, die Ereignisse übertrugen und somit ein ungewöhnlich starker öffentlicher Druck entstand. Hier, in der sogenannten „Schlacht um Zion“, erlitt das Ministerium für Staatssicherheit Ende 1987 seine erste große Niederlage. Heute spricht man von der ersten Niederlage, weil man weiß, was 1988 und 1989 folgte. Die Berliner von 1987 wussten das nicht. Für die politisch Interessierten unter ihnen aber häuften sich die Anzeichen für eine Veränderung - ohne dass man Art, Richtung oder Tempo erahnen konnte.

Es kann deshalb nicht häufig genug betont werden, dass 1987 vieles vorstellbar war, nur nicht ein baldiger Fall der Berliner Mauer. Bei aller unterschiedlicher Bewertung der Lage waren sich Ost und West 1987 wenigstens darüber einig: Ein Ende der deutschen Teilung käme, wenn überhaupt, in ferner, ferner Zukunft. Erst wenn man sich dies vergegenwärtigt, werden die Handlungen, Spannungen und Hoffnungen des Jahres 1987 verständlich, wie auch die spezifische Bedeutung mancher Worte. Da wäre etwa der Appell von Reagan an Gorbatschow, die Mauer niederzureißen: bestechend und kühn gerade wegen seiner Wirklichkeitsferne. Oder man denke an die zahlreichen Beteuerungen des Senats, dass Berlin eine Stadt sei und bleibe. Dies war 1987 weder ein Allgemeinplatz noch eine vorausschauende Ankündigung des baldigen Mauerfalls. Es ging vielmehr um die politische Entscheidung, auch gegen den Strom der Zeit an einer Vorstellung von Berlin festzuhalten, die vielen Zeitgenossen als historisch überholt galt. Gerade der Kontrast zur West-Berliner Alltagswirklichkeit machte 
das Einheitspathos damals so markant und teilweise auch umstritten. Substanzielle Veränderungen erwartete man dennoch auch in Regierungskreisen nicht, wie die Einschätzung eines Senatssprechers 1986 illustriert, der das kommende Jubiläum eine große Sache nannte, „vermutlich für Berlin das größte Ereignis bis zum Ende dieses Jahrhunderts“(38). Hoffen konnte man vieles, glauben aber kaum. Und ein paar Jahre später tanzten die Berliner auf der Mauer.

Und so war es auch nach dem überspannten, doppelten Stadtjubiläum. Trotz einiger aufregender Ereignisse hatten sich die meisten Zukunftserwartungen kaum verbessert. Im Gegenteil, Ende 1987 gab es überall Übermüdung und Skepsis - man hatte die ewige Selbstfeier satt, nicht nur im Osten, sondern auch im Westen. Mit einigem Unbehagen blickte man jetzt auf das Jahr 1988, denn dann sollte West-Berlin zu allem Überfluss auch noch Europas Kulturhauptstadt werden (im Volksmund „751-JahrFeier"). Versuchte die Inselstadt ihre Sinnkrise mit großen Festivals zu überdecken? So etwa kommentierte das Volksblatt Berlin und schaute Ende 1987 spöttisch in die Zukunft: 1987 Berlin-Jubiläum, 1988 Kulturhauptstadt, „für 1989 wird noch ein Anlaß zu feiern gesucht“"(39).

\section{Zusammenfassung}

Die Systemkonkurrenz in Berlin kreierte eine ganz besondere Feierkonkurrenz: Dreimal feierte Berlin im 20. Jahrhundert Geburtstag, jedes Mal unter anderen ideologischen Vorzeichen. Im Jahre 1937 beging die nationalsozialistische Reichshauptstadt das 700jährige Jubiläum Berlins. Folgerichtig gab es in der Mauerstadt 1987 eine doppelte 750-Jahr-Feier: einmal in der Hauptstadt der DDR und einmal in West-Berlin. Stets ließen sich im Gewand der Geburtstagsfeier politische Werte vermitteln: emotionale Bindungen, Freund-Feind-Bilder, Gemeinschaftserfahrungen. Der Beitrag gibt einen vergleichenden Überblick über die Selbstdarstellungen und fragt nach Beteiligung, Kritik und Tragweite der Festprogramme. Er zeigt, wie die Stadtgemeinschaften in der Herausbildung und Schärfung ihrer politischen Identität stets aufeinander bezogen blieben.

\section{Résumé}

La concurrence des systèmes à Berlin a créé une concurrence des jubilés. Au XXe siècle, Berlin fêta à trois reprises son anniversaire, à chaque fois sous des prémisses idéologiques différentes. En 1937, la capitale du Troisième Reich fêta son $700^{e}$ anniversaire; et, en 1987, il y eut deux organisations distinctes de festivités, dans la capitale de la RDA et à Berlin-Ouest. Ce fut l'occasion de transmettre des valeurs politiques en mettant en évidence des liens émotionnels, en articulant des images ennemis/amis, en soulignant l'expérience d'une communauté. Cet article se propose à travers une approche comparative de donner un aperçu d'ensemble des autoreprésentations auxquelles ces festivités ont donné lieu et s'interroge sur la participation aux événements, sur la critique et la portée du programme des festivités. Il montre aussi comment les deux villes, dans l'accentuation de leur identité politique, n’ont eu de cesse de se référer l'une à l'autre. 